\title{
Vidéoformation « orientée-activité » : quelles utilisations pour quels effets sur les enseignants?
}

\author{
Simon Flandin*, Serge Leblanc** \& Alain Muller*** \\ *Ifé-ENS de Lyon et Université de Clermont-Ferrand \\ **Université de Montpellier \\ ***Université de Genève
}

\section{Introduction}

Si l'usage de la vidéo en formation des enseignants ne constitue ni une pratique ni un domaine de recherche récents, le développement exponentiel des outils numériques et leur démocratisation ces dix dernières années a considérablement participé de la relance et de la diversification de ces pratiques et des moyens de les étudier (Gaudin \& Chaliès, 2012). Il en résulte une littérature abondante sur le sujet. L'un des concepts majeurs autour duquel elle s'organise est celui d'une «vision professionnelle ${ }^{1}$ » des enseignants qu'il s'agirait de développer par différentes modalités de vidéoformation. De nombreuses études récentes consistent à tester de façon quantitative ces modalités et leurs combinaisons comme des inputs : vidéo de soi vs vidéo d'un autre (Seidel, Stürmer, Blomberg, Kobarg, \& Schwindt, 2011), vidéo d'un collègue vs vidéo d'un inconnu (Zhang, Lundeberg, Koehler, \& Eberhardt, 2011), vidéo d'un cours en sciences humaines vs vidéo d'un cours en sciences physiques / mathématiques (Blomberg, Stürmer, \& Seidel, 2011), etc. Les outputs sont quant à eux des indicateurs objectivant cette vision professionnelle et tirés de modélisations extrinsèques du travail expert, à partir desquels les enseignants en formation (EEF par la suite) sont évalués après avoir suivi la vidéoformation. Ce mode de recherche présente aux moins deux limites majeures : 1) l'activité réelle des enseignants engagés dans ces dispositifs est faiblement prise en compte (Gaudin \& Chaliès, à paraître ${ }^{2}$ ), et tout se passe comme si un bon dispositif de formation pouvait se résumer à une simple computation de modalités présumées isolément efficaces, indépendamment des caractéristiques et engagements des utilisateurs (Flandin \& Ria, 2014d) ; 2) les effets sur l'activité réelle de travail ne sont pas étudiés, et on pourrait être tenté de voir la vision professionnelle comme prédictive de la capacité à enseigner (Kersting, Givvin, Sotelo, \& Stigler, 2010).

\footnotetext{
${ }^{1}$ La vision professionnelle est définie comme la capacité de l'enseignant à remarquer et à interpréter les éléments objectivement pertinents des interactions en classe (Sherin, 2001).

${ }^{2}$ Ces auteurs montrent que, dans ces études, l'activité des EEF est souvent réduite à des composantes d'attention sélective et de réflexion, voire même à la seule tâche, c'est-à-dire à ce qui leur est demandé de faire dans le dispositif.
} 
Nous inscrivant dans une approche située de l'activité, de nature sémiotique et phénoménologique (Peirce, 1978 ; Theureau, 2004), nous pensons au contraire que l'efficacité d'un dispositif réside dans la forme de médiation qu'il instaure dans le rapport qu'entretiennent les enseignants formés avec leur travail, c'est-à-dire l'activité que ce dispositif tend à générer chez eux en situation de vidéoformation et les transformations réelles ou potentielles de leur activité d'enseignement. Or, nous postulons que cette médiation n'existe pas avant d'être produite par l'activité se faisant, et ne peut se déterminer avant et indépendamment d'elle (Flandin \& Ria, 2012 ; Linard, 1989 ; Steiner, 2010). À ce titre, nous menons des travaux de recherche-conception qui articulent de manière systématique : a) l'analyse de l'activité réelle des EEF au travail, b) l'analyse de leur activité réelle en formation, et c) l'élaboration continue de principes, d'artefacts et de dispositifs de vidéoformation. En cherchant à répondre à des préoccupations ergonomiques, ce modèle organise un programme de recherche (Flandin \& Ria, 2014a; Leblanc \& Ria, 2014 ; Leblanc, Ria, Dieumegard, Serres, \& Durand, 2008 ; Ria \& Leblanc, 2011) assez innovant en regard des limites évoquées précédemment : 1) il permet de rendre compte de l'expérience vécue par les EEF dans les dispositifs et d'identifier les processus qui contribuent à leur engagement et à leur apprentissage ; 2) il permet de comprendre l'activité réelle des EEF et d'indexer à ses modes de transformation de nouvelles modalités de vidéoformation, sans les réduire à l'acquisition de savoirs prédéfinis.

Le but de cet article est de faire une synthèse des résultats obtenus dans ce programme au terme d'études menées sur l'utilisation de sa réalisation majeure: la plateforme Néopass@ction (Ria \& Leblanc, 2011). À partir de ces exemples, il s'agit de rendre compte des effets potentiels de la vidéo sur l'activité en formation et sur le développement professionnel, et de proposer des pistes pour la conception de situations de vidéoformation. Nous présentons dans une première partie la structure du programme de recherche-conception en vidéoformation des enseignants. Dans une seconde partie, nous comparons de façon thématique et synthétique les résultats des études menées et leurs implications pour la conception de formation. Enfin, nous proposons dans une troisième partie un méta-modèle d'analyse anthropo-sémiotique de l'expérience de vidéoformation, avant de conclure dans une perspective technologique.

\section{Un programme de recherche empirique et technologique en vidéoformation des enseignants}


Nos travaux s'inscrivent dans une épistémologie de l'expérience qui postule l'indissociabilité de l'acteur et du milieu non pas dans lequel il vit, mais par le moyen duquel il vit (Dewey, 1993 ; Varela, 1989). Les deux se co-déterminent et se co-développent au fil de situations, entendues comme états relationnels dynamiques vécus par l'acteur et donnant lieu à expérience. L'activité correspond à l'engagement de l'acteur au milieu, dans des situations instables qu'il cherche à rééquilibrer, relativement à des normes de viabilité qui lui sont propres. Cette activité est cognitive, c'est-à-dire production de signes, le signe étant conçu dans ce cadre comme une relation triadique ${ }^{3}$ entre un objet du milieu, son représentamen (la façon dont le perçoit, le «délimite » l'acteur) et son interprétant (la signification que lui attribue l'acteur). Notre méthode de recherche vise donc qualitativement à rendre compte de cette production de signes en conduisant des entretiens avec l'acteur, qui est à cette occasion «dynamiquement resitué » dans l'action à l'aide de traces vidéo de son activité (« autoconfrontation », Theureau, 2010).

Dans cette approche, la formation consiste à la fois à provoquer des ruptures dans l'équilibre relationnel de l'acteur à son milieu, et à la fois à lui permettre de le rétablir en encourageant et décourageant certaines actions (Durand, 2008). Nos travaux consistent donc à identifier empiriquement les conditions dans lesquelles des expériences vécues en situation de vidéoformation tendent à développer l'activité des EEF vers une plus grande viabilité de leurs situations de travail, c'est-à-dire en particulier vers une plus grande efficacité dans la poursuite des objectifs auto-prescrits et prescrits par l'institution, et vers une plus grande économie de soi. L'analyse de ces résultats nous permet : a) de proposer des principes de conception (activités à favoriser, nature des consignes et des extraits vidéo, espaces et temporalités à investir, etc.) ; b) de mettre à disposition des EEF et de leurs formateurs des outils et dispositifs (notamment numériques et en ligne) ; et c) d'améliorer continuellement ces productions. Ce volet technologique engendre des situations de formation inédites qui peuvent à nouveau être investiguées de façon empirique, et ainsi de suite.

Dans le cas de la plateforme Néopass@ction, le programme agence les phases de rechercheconception suivantes :

1. À partir d'un observatoire du travail enseignant, des situations de travail typiquement problématiques ont été identifiées et les dispositions à agir typiquement mobilisées par les enseignants pour y faire face ont été modélisées (Ria, 2009);

\footnotetext{
${ }^{3}$ Une relation triadique signifie que chacune des instances du signe n'existe qu'en tant qu'elle est en relation avec les deux autres, donc que toute transformation d'une des instances transforme les autres.
} 
2. Ces couples situations-dispositions ont été exemplifiés par des enregistrements vidéo de classe et d'entretien, agencés entre eux dans le but de constituer des artefacts vidéo pouvant être utilisés dans des situations de formation professionnelle (Ria \& Leblanc, 2011) ;

3. Ces situations de formation ont constitué autant de tests itératifs permettant d'identifier les points faibles de ces artefacts et de les améliorer progressivement: ces boucles de conception ont abouti à un prototype de plateforme (Ria \& Leblanc, 2011) ;

4. La plateforme a fait et continue de faire continuellement l'objet de «simulations participatives » auprès de différents publics d'EEF (formation initiale, année de stage, formation continue), dans différents formats (formation à distance, formation en présentiel, formation hybride) et selon différentes modalités (autoformation, collectif intraétablissement, dyade avec un formateur, travail dirigé en petit groupe, cours magistral en grand groupe...) La conception est ainsi continuée dans l'étude de ces diverses utilisations et la plateforme améliorée : nouvelles ressources, création de scénarios pédagogiques, élaboration d'artefacts complémentaires... (Flandin \& Ria, 2014a; Leblanc \& Ria, 2014).

Chaque phase spécifie conjointement de nouveaux objets de recherche et de nouveaux objets de conception. Cet article analyse les résultats des études menées dans les simulations participatives décrites en phase 4 .

\section{Synthèse des études}

Cette partie vise à synthétiser les résultats d'études basées sur des recueils de données qualitatives portant sur l'utilisation de la plateforme Néopass@ction, et non à rendre compte de leurs dimensions méthodologiques et empiriques ${ }^{4}$.

Le premier niveau de recueil a systématiquement consisté en l'enregistrement vidéo de traces de l'activité déployée par les EEF dans une situation-cible de vidéoformation (Leblanc, 2014a ; Leblanc \& Sève, 2012 ; Lussi Borer \& Muller, 2013, 2014a, 2014b, à paraître ; Ria \& Leblanc, 2012). Un second niveau de recueil a consisté en l'enregistrement vidéo d'entretiens d'autoconfrontation (Theureau, 2010) menés a posteriori avec les EEF (Flandin \& Ria, 2014a, 2014b; Leblanc \& Blanes, 2014).

\footnotetext{
${ }^{4}$ Pour plus de détails, les auteurs renvoient le lecteur aux articles cités en référence.
} 
Ces études ont la particularité de chercher à rendre visibles non seulement les effets transformatifs de l'activité menée sur la plateforme, mais plus encore les processus de transformation eux-mêmes. Ces processus de transformation de l'activité - correspondant à des déséquilibrages et rééquilibrages de la relation de l'acteur et de son milieu - peuvent être saisis à trois niveaux :

1. Le niveau de la «mise en relation» de l'activité visionnée et de l'activité, ou de l'expérience, de celui (ou de ceux) qui travaillent sur la plateforme. On tente de répondre à la question: qu'est-ce qui rend possible, dans le dispositif, les transformations de l'activité?

2. Le niveau des processus de transformation eux-mêmes. On tente de répondre à la question : de quoi sont composés ces processus de transformation?

3. Le niveau des dispositifs-environnements de formation. On tente de répondre à la question: quelles sont les conditions permettant de susciter au mieux ces processus de transformation?

Ces trois niveaux ne sont pas à comprendre comme renvoyant à des processus séparés, mais comme des focalisations particulières sur un même et unique processus de transformation : 1) focalisation plutôt d'ordre topologique dans le sens où il s'agit de rendre compte de l'espace phénoménologique et sémiotique suscité par le dispositif; 2) focalisation plutôt chronologique au sens où il s'agit de rendre compte du déploiement temporalisé de cet espace phénoménologique et sémiotique; 3) focalisation écologique au sens où cet espace phénoménologique et sémiotique est saisi à l'aune de l'environnement au sein duquel il se déploie temporellement.

\section{Mise en relation de l'activité visionnée et de l'activité / expérience de l'utilisateur}

Les études à focalisation topologique se déploient sur deux axes, l'un plutôt phénoménologique visant à décrire une expérience vécue, l'autre plutôt sémiotique visant à identifier les divers niveaux de significations attribués à cette expérience vécue.

Le processus phénoménologique le plus documenté est un processus mimétique consistant dans la mise en lien de l'activité de l'utilisateur et de l'activité visionnée à l'aune de leur proximité, de leur «mêmeté ${ }^{5}$ ». L'utilisateur fait l'expérience de l'activité d'un autre comme si c'était la sienne, ce qui tend à l'immerger spatialement et temporellement dans l'activité

\footnotetext{
${ }^{5}$ Montrant des situations d'enseignement prototypiques, la plateforme Néopass@ction tend à susciter ces expériences de proximité ou de « mêmeté ».
} 
visionnée (Citton, 2012), à vivre celle-ci par procuration et à rejouer la sienne, ceci à travers des processus de synchronisation, de création d'attentes, et d'anticipation (Leblanc, 2014a).

Ce processus mimétique peut être saisi comme une mise en lien de la relation entre activité visionnée et activité de l'utilisateur sous le double aspect d'un point de vue égocentré - je vis ce que je vois comme si c'était moi qui le vivais - et allocentré - je me mets à la place de l'autre. Le passage du point de vue égocentré au point de vue allocentré peut s'ouvrir vers un troisième point de vue, moins lié au vécu, plus générique et décontextualisé (Ria \& Leblanc, 2011).

Cette immersion dans l'activité visionnée peut aussi prendre l'aspect d'une expérience fictionnelle (Durand, 2008 ; Zaccaï-Reyners, 2005) dans laquelle de nouveaux possibles pour sa propre activité réelle émergent sur la base d'éléments significatifs identifiés dans l'activité visionnée, engendrant tout un jeu de faire comme si (Ria \& Leblanc, 2011).

En ce qui concerne les travaux (Lussi Borer \& Muller, 2014a) ancrés dans une analyse sémiotique peircienne (Peirce, 1978), ils montrent que les relations entre les utilisateurs de la plateforme et l'activité visionnée peuvent être classées en deux grandes classes de signes, composée chacune de cinq signes :

Les signes de la première classe expriment la relation à l'activité visionnée comme :

1. Réaction simple : hochement de tête, sourire...

2. Description d'événement : «les élèves arrivent tranquillement... »

3. Interprétation de l'activité : « elle regarde l'élève pour lui signifier de se calmer ».

4. Explication de l'activité : « si la classe est calme, c'est parce qu'il y a des habitudes qui ont été construites ».

5. Appréciation de l'activité : «c'est bien... ça marche »

Les signes de la seconde classe expriment la relation de l'activité visionnée à une autre activité (très souvent celle des EEF) comme :

1. Description de sa propre activité.

2. Mise en parallèle d'événements : «moi aussi j'ai des classes agitées ».

3. Mise en parallèle d'intentions : « moi aussi j'essaye de motiver les élèves ».

4. Confrontation de deux activités : « elle laisse entrer les élèves comme ils veulent... moi je dis non... chez moi on les regroupe ».

5. Évaluation de l'activité : «mettre les élèves en rang... pour des élèves de cet âge ce n'est vraiment pas pertinent $»$. 
Bref, la confrontation des EEF à l'activité filmée d'un pair semble mettre en mouvement un processus de sémiotisation, et de l'activité visionnée et de leur propre activité, potentiellement porteur de transformations de cette dernière.

\section{Processus de transformation}

De manière très générale, ces études identifient des processus de transformation des connaissances professionnelles et du sens de l'expérience des EEF aboutissant à des modifications potentielles ou réelles de leur activité.

Bien que selon leur référent conceptuel, les études «thématisent» différemment ces processus, un accord semble s'être établi sur un double constat :

- on a affaire à des transformations silencieuses (Jullien, 2009) qui, à première vue, peuvent paraître «modestes» mais qui, dans la mesure où elles tendent à se propager progressivement, de proche en proche, en intégrant les oppositions traversant l'activité ${ }^{6}$, sont porteuses de développements importants et pérennes (Leblanc, à paraître).

- ces transformations se font toujours par intégration continue du nouveau au déjà-là, dans un tissage de modifications et de continuations (Jullien, 2009 ; Leblanc, 2014a), ou encore par actualisation d'un potentiel resté « caché » dans l'activité (Lussi Borer \& Muller, 2013).

Ces transformations silencieuses opèrent à trois niveaux en interaction - expériences de navigation, rapports à sa propre activité, normes d'action - et sont soutenues par trois processus d'imagination, de sémiotisation et d'individuation.

Ont été premièrement identifiées diverses modalités d'usage de la plateforme (Flandin \& Ria, 2014c). Un premier type de navigation consiste à «butiner» de manière exploratoire sur la plateforme sans que les contenus visionnés ne suscitent d'expérience réellement significative. Un deuxième type d'expérience consiste en un amorçage observationnel dans lequel l'acteur reconnaît dans la situation visionnée des similarités avec des situations vécues du point de vue de leur non-viabilité, ce qui tend à engendrer un processus d'enquête sur les possibilités de rendre ce genre de situation viable. Dans un troisième type d'expérience, l'acteur perçoit la situation visionnée comme étant viable, et comme lui offrant des pistes de transformation possibles de sa propre activité.

À travers ces expériences contrastées peuvent émerger des sentiments d'insatisfaction face à sa propre activité (Flandin \& Ria, 2014b). Cette insatisfaction peut se manifester de deux manières. On observe premièrement que le visionnement d'un film peut «révéler»,

\footnotetext{
${ }^{6}$ Voir à ce propos le concept de transduction développé par Simondon (2005).
} 
« remobiliser» ou «actualiser» une insatisfaction déjà là (mais de façon tacite, infraconsciente) concernant sa propre activité. Deuxièmement, une insatisfaction peut naître au moment où une activité visionnée est perçue comparativement comme étant meilleure que la sienne, c'est-à-dire plus viable.

Ces émergences d'insatisfactions sont potentiellement porteuses de processus de renormalisation, soit de transformation des règles à suivre dans l'action, processus dont on peut repérer trois modalités ${ }^{7}$. Dans la première modalité (Lussi Borer \& Muller, 2014b), le visionnement d'une situation d'enseignement du même type qu'une situation vécue suscite chez les EEF un double mouvement d'explicitation de règles implicites et d'élaboration de conflits entre les règles personnelles et les règles du milieu (institution et attentes des pairs), ce qui en dernier lieu leur permet de chercher une « sortie » de ces conflits de règles.

Dans la seconde modalité (Lussi Borer \& Muller, 2013), la renormalisation prend sa source dans l'articulation d'un double geste de comparaison inter-activité et intra-activité : le visionnement de plusieurs films montrant des situations d'enseignement de même type permet de saisir des différences entre activités, mais aussi des différences possibles dans une même activité. L'articulation de ces deux différences consiste à aller chercher ce qu'il serait possible de transformer dans une activité, dans ce qui se passe réellement dans une autre activité.

Dans la troisième modalité, la renormalisation s'appuie sur une activité de multiréférenciation à différentes instances de la plateforme, ainsi qu'à la mise en lien de celles-ci dans un processus hélicö̈dal d'élaboration de l'activité (Lussi Borer \& Muller, à paraître). Ces différentes instances sont: l'activité de classe visionnée "en premier lieu », le vécu professionnel de l'enseignant visionné, d'autres activités de classe visionnées, d'autres vécus professionnels visionnés, les témoignages d'autres enseignants visionnés, la propre activité de l'enseignant visionnant les films, des activités « abstraites » de référence considérées comme « bonnes» ou « réussies», des activités «abstraites» de référence considérées comme typiques chez les débutants. Le jeu qui s'instaure par la mise en dialogue de ces différentes instances est à l'origine de déplacements tant cognitifs (transformation dans la compréhension de l'activité d'enseignement) que normatifs (transformation des règles d'action que les EEF se donnent).

Ces trois niveaux de transformation sont soutenus par trois processus liés « d'arrière-plan » de sémiotisation, d'imagination et d'individuation.

\footnotetext{
${ }^{7}$ Notons que ces trois modalités ne sont pas exclusives les unes des autres, qu'elles peuvent se conjuguer ; leur distinction doit être prise comme plus analytique que factuelle.
} 
Une analyse sémiotique (Lussi Borer \& Muller, 2014a) montre que les EEF confrontés à une activité filmée tendent à produire des trames sémiotiques en parcourant divers niveaux de sémiotisation, avec pour conséquence que ce qui est «dit» dans un premier temps à un certain niveau peut être modifié dans un deuxième temps suite au passage par d'autres niveaux. Par exemple, ils passent de l'appréciation à la description via une interprétation de l'activité, pour revenir à une nouvelle appréciation, différente de la première. Bref, la traversée des trames sémiotiques aboutit à un processus de déconstruction-reconstruction de l'activité visionnée porteur de renormalisations.

Ces sémiotisations semblent s'articuler à un triple processus d'imagination : la confrontation à l'activité visionnée peut susciter l'émergence d'images-souvenirs réactivant une expérience vécue, mises en lien par écho à des images-perçues de l'activité d'un tiers, lien sur la base duquel peuvent émerger des images-esquisses profilant une activité future (Leblanc, 2014a). Ainsi, les processus de sémiotisation et d'imagination s'ancrent temporellement dans « l'articulation et la relation entre le passé et l'avenir »(Leblanc, à paraître), participant en cela d'un processus d'individuation (Simondon, 2005), soit de « transformation de l'individu en tant que demeurant toujours inachevé et d'objectivation de l'expérience, de la relation à soi-même et au monde extérieur » (Leblanc, à paraître).

\section{Environnements de formation}

Les résultats des études concernant les conditions les plus susceptibles de favoriser des processus de transformation se répartissent sur les axes suivants :

1. L'axe navigation autonome vs navigation médiée par un formateur.

2. L'axe réflexion-analyse vs vécu-engagement.

3. L'axe complétude transformative vs incomplétude transformative.

Les recherches centrées sur la comparaison de navigations autonomes et de navigations accompagnées (Flandin \& Ria, 2014a) créditent le principe selon lequel l'accompagnement d'un formateur augmente la probabilité que le travail sur l'activité d'autrui suscite une expérience significative, et ceci d'autant plus que l'enseignant en formation n'a pas (ou peu) d'expérience professionnelle.

Mais en quoi l'accompagnement du formateur doit-il consister ?

Une des premières conditions concerne la position du formateur qui a avantage à être empathique et «symétrique » (non en surplomb), à l'écoute des besoins des enseignants en formation. 
Sur cette base empathique, l'activité du formateur peut simplement consister à aider les EEF à « élaborer une problématique de navigation » (Flandin \& Ria, 2014a), à expliciter ce que le visionnement de films suscite comme réactions chez eux, à identifier leurs propres croyances et modes d'agir à travers ceux des autres, à imaginer des nouvelles manières d'agir (Leblanc, 2014a), ou encore à élaborer des conflits de normes susceptibles de déboucher sur des renormalisations.

En ce qui concerne le deuxième axe, les études semblent montrer que ce n'est pas l'analyse et/ou la réflexion sur les situations visionnées (et les situations vécues) qui permet fondamentalement d'engager des processus de transformation, mais bien plutôt le fait que le visionnement d'autrui suscite un « revécu » de sa propre activité dans toutes ses dimensions, cognitives et affectives. Bref, il s'agit de mettre en place un environnement directement adressé au travail de l'enseignant et non à l'analyse de celui-ci (Flandin \& Ria, 2014c; Leblanc \& Blanes, 2014). Néanmoins, cela ne signifie pas qu'il faille simplement laisser les EEF revivre les situations et ne pas mettre en place des conditions de «prise de distance ». Premièrement, le «ré-engagement» dans une activité vécue semble être une condition nécessaire d'entrée dans un travail d'élaboration et de transformation de l'activité, mais non suffisante : si ce « ré-engagement» apparaît être un passage obligé auquel il s'agit de donner toute sa place, il ne peut cependant prendre son entière signification et susciter du changement qu'à l'aune de sa conjugaison avec un certain « dégagement analytique » permettant aux EEF de «s'interroger sur leurs croyances, convictions, dispositions à agir, façons de faire non ou peu questionnées afin de construire les problèmes professionnels et d'envisager de nouveaux champs de possibles » (Leblanc et Blanes, 2014). Deuxièmement, l'accès à sa propre activité via celle de l'autre ne se fait pas forcément «naturellement», tant le visionnement de films tend à susciter en premier lieu un regard extrêmement normatif - ancré paradoxalement souvent dans une normativité institutionnelle que les EEF rejettent par ailleurs - brouillant l'accès à une compréhension de la logique interne de l'activité. Il peut être ainsi utile, voire nécessaire, d'exercer une «contrainte » sur ce regard, en essayant de le rendre momentanément plus interprétatif et/ou plus descriptif, ce qui permet dans un deuxième temps de revenir dégagé de jugement à sa propre activité (Lussi Borer \& Muller, 2014a, 2014b).

Le troisième axe s'attelle au problème de «l'extension spatio-temporelle» des environnements de formation. Ceux-ci visent évidemment bien in fine une transformation de l'activité réelle des EEF. Mais faut-il aller jusqu'à intégrer celle-ci dans le dispositif - c'est-àdire instituer des allers-retours entre moments d'élaboration de l'activité et de ses 
transformations possibles via des alloconfrontations hors classe et moments de mise en œuvre en classe de ces transformations? Certaines recherches (Lussi Borer \& Muller, 2014b) arrivent à la conclusion que les «boucles transformatives» doivent, pour pouvoir être véritablement formatrices, aller jusqu'à la mise en œuvre. D'autres recherches semblent aller dans le sens d'une « complétude transformative » des environnements de formation organisés autour de l'unique espace d'alloconfrontation - cet espace est transformateur en lui-même et in situ - dans la mesure où «la conscientisation d'anticipations représentées sous formes d'images et des conséquences tirées des expériences passées en lien avec la situation seront des ressources mobilisables en cours d'action » (Leblanc, 2014a, p. 23) en ce qu'elle constitue « par avance une expérience » (Simondon, 2008, p. 277).

\section{Méta-modèle d'analyse de l'utilisation de films issus du travail réel en formation}

Cette partie développe une réflexion relative aux apports du voir pour le faire et aux conditions à mettre en œuvre pour favoriser un engagement pro-actif en formation sous-tendu par des questions de terrain, et pour inciter l'expérimentation de façons de faire inspirées par la confrontation à des films issus du travail réel. Elle se situe à un niveau plus général que la synthèse des études faite précédemment et présente un méta-modèle qui analyse les dimensions anthropologique et technologique de l'activité humaine médiatisée.

La vidéo comme " objet temporel" pour s’immerger dans, simuler, imaginer le travail en formation

La possibilité technologique d'enregistrer des vidéos numériques, de les stocker, de les agencer facilement et les diffuser quasi-immédiatement en présentiel ou à distance révolutionne potentiellement les pratiques de formation professionnelle qui jusque-là étaient contraintes de saisir-prélever en une fois et dans l'instantanéité de la situation de terrain un certain nombre d'informations, avec la difficulté voire l'impossibilité de revenir sur un événement passé. Les enregistrements vidéo possèdent des propriétés spécifiques de conservation des relations entre des éléments de la réalité et de la dynamique singulière de la situation qui les distinguent des deux principales méthodes de saisie-restitution d'une situation observée (Veillard, 2012) : la prise de notes ouverte et l'utilisation d'une grille d'observation. Le film, nous dit Sensevy (2012, pp. 6-8), est « une sorte d'analogue de l'action », mais il n'est pas l'action; il ne représente pas le réel, il « témoigne » d'une réalité. 
Il se caractérise principalement par deux dimensions : d'une part sa «densité », due à sa « représentation analogique » qui fait que l'on peut en faire de très nombreuses descriptions et d'autre part, son « isomorphisme structural avec la réalité » sur le plan spatio-temporel qui permet d'appréhender le détail des gestes, des déplacements, des distances ainsi que le flux temporel de la pratique (Sensevy, 2012). Mais des EEF confrontés au même enregistrement vidéo ne restituent jamais ou rarement la même vision de la situation, ce qui en fait un levier de formation à condition d'en comprendre les principales raisons. Les différentes possibilités de signifier l'activité visionnée, décrites précédemment en deux grandes classes de signes (Lussi Borer \& Muller, 2014a), renforcent cette idée.

Pour expliquer ce phénomène, nous nous appuyons sur les travaux de Stiegler (2010) qui, en reprenant les travaux d'Husserl sur les objets temporels, distinguent trois systèmes de rétention différents utiles pour comprendre ce qui se joue lors du visionnage d'enregistrements vidéoscopiques. Un enregistrement vidéo constitue un « objet temporel audiovisuel» qui se caractérise par «son écoulement : la condition de son apparition à ma conscience, c'est sa disparition; il disparaît à mesure qu'il apparaît » (Stiegler, 2010, p. 75). Comme nous sommes nous-mêmes des êtres essentiellement temporels toujours tournés vers le futur et l'anticipation, cet objet temporel qu'est la vidéo possède la particularité d'avoir une structure d'écoulement semblable à celle de notre conscience : elle génère des processus de synchronisation et de création d'attentes.

La rétention primaire constitue le présent. Elle articule dans la perception même de l'objet temporel le présent, un tout-juste-passé et une anticipation sur ce qui va suivre immédiatement. Ce qui permet de donner du sens à ce que l'on regarde (un film) ou à ce que l'on écoute (un commentaire) est dû au fait que l'on ne voit pas une succession d'images ou n'entendons pas une succession de fréquences sonores mais que notre conscience crée un « rapport temporel de continuité » entre ces images ou ces sons (Steiner, 2010). L'attitude d'un enseignant en attente les bras croisés n'a d'intelligibilité que rattaché à l'instant t-1 ou t$\mathrm{n}$ qui le précède et prend du sens dès que l'on y ajoute le geste tout-juste-passé (p. ex. les remarques adressées à des élèves agités). Si le plan retient, conserve le geste précédent, il est en même temps tourné vers la scène à venir, il protient et crée une attente sur le plan suivant (p. ex. les élèves vont-ils se calmer ou s'exciter encore plus ?). Cette double caractéristique de rétention-protention permet de créer en formation des situations fictionnelles dans lesquelles les acteurs vont s'immerger mimétiquement et se plonger dans des horizons d'attente faits à la fois de connu et d'inattendu. Ces immersions à la fois temporelles et spatiales favorisent l'engagement des formés dans la posture du faire comme si en leur permettant de se glisser 
dans un univers fictionnel. Recourir à l'imaginaire est donc une condition indispensable pour atteindre le réel (Schaeffer, 1999). Il participe notamment de l'invention des possibles dans le double geste de comparaison inter-activité et intra-activité décrit par Lussi Borer et Muller (2013), ainsi que de l'imagination de leurs combinaisons possibles dans les processus hélicoïdaux d'élaboration de l'activité décrits par ces auteurs (à paraître).

La rétention secondaire constitue le passé et relève du souvenir. Elle correspond aux « savoirs » accumulés par nos expériences passées qui constituent autant d'horizons d'attente, c'est-à-dire de protentions. En lien avec le plan vidéo visionné à l'instant t (l'enseignant en attente, les bras croisés), sont convoqués, mobilisés ses propres expériences relatives à une situation de classe perturbée par quelques élèves et à la manière dont on les a gérés. Lorsque l'on demande à un groupe d'EEF de décrire ce qu'ils ont vu de l'activité d'un collègue, on peut constater une différence entre leurs rétentions primaires. Une simulation réactive des états vécus dans des expériences antérieures aussi bien sur les registres de la perception, de l'action, des émotions et des pensées. Notre perception du monde repose sur la résonance en nous de «mimismes gestuels» (Citton, 2012). Ainsi, durant la situation de simulation amorcée par l'observation de vidéos de classe, le voir est un voir « en écho », proche de ce que Ricœur nomme « voir comme » $(1990$, p. 14) pour qualifier les processus métaphoriques et mimétiques comme anthropologiquement constitutifs de l'expérience humaine, et non un voir pour «imiter des aspects formels et superficiels» du comportement de l'enseignant (Leblanc, 2014a, p. 158). Ce dernier se synchronise toujours avec des connaissances, des expériences de classe vécues plus ou moins proches de la situation visionnée, qui sont mises en relation et qui permettent de créer des échanges interactifs de type mimétiques (postures, gestes, déplacement, communication, silence, intonation...) entre le spectateur-formé et l'enseignant à l'écran. Cela explique, comme l'ont montré Flandin et Ria (2014c), que les enseignants ne retiennent pas tous les mêmes gestes comme significatifs ni la même articulation gestes passé-présent-futur. Cette différence s'explique parce que ce sont les rétentions secondaires qui déterminent les rétentions primaires; autrement dit, ce que l'on voit est toujours filtré par nos expériences. Un enseignant débutant n'ayant jamais vécu directement ou indirectement (p. ex. à travers des situations de vidéoformation) une situation avec des élèves rétifs ne va pas retenir les mêmes éléments significatifs lors d'un premier visionnement que celui qui en a déjà fait l'expérience. Cela explique également le fait qu'émerge ou non un sentiment d'insatisfaction envers sa propre activité (Flandin \& Ria, 2014b). 
Cette articulation entre rétention primaire et secondaire joue un rôle essentiel en formation car elle ouvre des espaces d'anticipation et de controverses professionnelles indexés à des situations typiques. Elle montre ainsi l'intérêt de constituer une expérience de classe élargie, directement (à travers des situations réelles de classe) et indirectement (à travers des situations de simulation) pour étoffer et enrichir la rétention secondaire, déterminante dans la manière de lire l'activité première. Les rétentions tertiaires proprement mnémotechniques constituées par ces environnements vidéo numériques génèrent des effets en partie contrôlables non possibles jusqu'alors en permettant de faire émerger : a) des différences de rétention primaire entre les acteurs mais aussi entre un premier, deuxième, troisième visionnement, chaque nouveau visionnement générant de nouvelles interprétations ; b) des différences entre rétention primaire et secondaire en créant des scénarios pédagogiques orientés soit vers les protentions (p. ex. à la place de cet enseignant à ce moment-là que feriez-vous ?), soit vers les rétentions secondaires (p. ex. quelles expériences personnelles directes ou indirectes évoquent cet épisode de classe ?). Créer les conditions favorables à un engagement pro-actif en formation susceptible de favoriser un tissage de modifications et de continuations (Leblanc, 2014a) passe par la mise en place d'un environnement adressé directement au travail de l'enseignant. Deux principes de conception sous-tendent particulièrement un tel environnement : a) favoriser et entretenir la connexion entre la phase d'observation-analyse de l'activité visionnée et les intentions d'exploitation plus ou moins implicites des EEF lors du visionnage ; et b) donner accès au vécu des enseignants filmés afin de prendre en compte les dimensions invisibles de l'activité et de reconstruire sans les séparer les registres de l'action, des émotions, des perceptions et cognitions.

\section{Voir le « travail réel " comme un processus de renormalisation de l'activité d'enseignement}

La plateforme Néopass@ction offre une possibilité d'accès direct et minimalement médiatisé au travail des enseignants en donnant à voir et en montrant, à travers des films, des ambiances, des micro-gestes, des dynamiques interactionnelles et des configurations d'événements dans leur état brut, ce que ne permettent pas les autres dispositifs d'analyse de pratiques ayant recours à la narration ou à l'écriture, qui restituent le travail comme un objet déjà structuré par le langage et ainsi déjà «trop » normé (Lussi Borer \& Muller, 2014a ; Quéré, 1993). Dans cette perspective, le travail réel, objet de visionnement et d'analyse en formation, joue le rôle d'une médiation entre les normes conscientes ou inconscientes, formelles ou informelles, personnelles ou collectives qui irriguent le travail à différents niveaux et les conditions de mise en œuvre de celles-ci qui s'expriment en fonction des 
épistémologies en termes de règles de métier, de savoirs pour enseigner, de savoirs tacites, « d'habitus professionnel »... (Lussi Borer \& Muller, 2014a).

La conception des dispositifs de formation professionnalisants peut être pensée à partir de deux visions contrastées de la modélisation de l'activité enseignante : a) soit elle est pensée comme un produit achevé qui est à atteindre par les enseignants débutants en appliquant les prescriptions institutionnelles, des savoirs scientifiques et/ou professionnels, l'efficacité étant alors conçue comme dépendante de cette application; b) soit elle est appréhendée comme un processus dynamique produisant dans l'action des «compromis opératoires » qui combine d'une part «ce qu'on demande » de faire au sujet à partir des prescriptions à la fois « descendantes » et « remontantes ${ }^{8} »$ et d'autre part de « ce que ça lui demande » pour réaliser de tels compromis, notamment au regard de sa propre histoire et de ses dispositions à agir du moment (Saujat, 2010), l'efficacité étant alors appréhendée sur la base de critères à la fois objectifs et subjectifs.

En envisageant l'apprentissage comme la conséquence de la transmission d'une représentation adéquate d'un monde extérieur prédéterminé qu'il faudrait réduire et reconstituer dans une situation de formation, il n'est pas possible d'appréhender ni les décalages avec les expériences vécues des enseignants débutants, ni les dilemmes et tensions professionnelles qui sont au cœur du métier. Se former professionnellement ne peut donc se réduire à s'approprier des savoirs, des règles prédéfinies et prescrites par un formateur dans un contexte de formation institutionnalisé. Il s'agit plutôt, sur la base des différentes expériences vécues, de faire-émerger dans un «environnement capacitant ${ }^{9} »$ des questions dont la pertinence est dictée par les significations construites par l'acteur lui-même. En visant une formation au travail réel, un «environnement capacitant» doit posséder un caractère énigmatique générateur de doutes, de questionnements favorables au déclenchement d'un processus d'enquête permettant de passer de l'indétermination à une détermination progressive (Dewey, 1993) par la mise en relation de « la triade : travail prescrit / conditions de mises en œuvre / travail réel » (Lussi Borer \& Muller, 2014a).

Les apprentissages ici et maintenant «de modes d'action nouveaux issus du patrimoine culturel professionnel » (Durand, 2008, p.100) s'articulent avec le développement du sujet ;

\footnotetext{
${ }^{8}$ La notion de prescriptions remontantes permet de prendre en compte ce qui est peu prescrit et ce qui « peut "remonter" de la matière travaillée» (Daniellou, 2002). Par exemple, les élèves qui ne comprennent pas une notion importante du programme s'opposent à la prescription descendante de devoir réaliser tout le programme défini par les textes pour tel niveau de classe dans l'année scolaire.

${ }^{9}$ Un « environnement capacitant » est conçu pour favoriser la mise en œuvre effective des capacités des acteurs. Les concepteurs de ces environnements cherchent à déterminer les conditions favorables et les « facteurs de conversion » du potentiel d'action des acteurs en possibilité réelle (Falzon, 2013).
} 
nous le définissons à un niveau typique-générique et sur du temps plus long comme «la transformation de son répertoire d'actions » (Durand, 2008, p. 100), ou de ses « dispositions à agir ", c'est-à-dire de ses propensions ou tendances à agir d'une certaine manière dans des circonstances déterminées (Lahire, 1998 ; Leblanc, 2014b). Pour transformer les dispositions à agir qui possèdent une certaine stabilité grâce à une forme d'économie et d'efficacité pour les personnes qui les mobilisent, il est nécessaire d'enclencher un processus de renormalisation (Lussi Borer \& Muller, 2013; Schwartz, 2007), c'est-à-dire d'appropriation et / ou de renouvellement de règles éprouvées dans l'action et dont la viabilité a été testée par soi-même et / ou d'autres. En confrontant les acteurs à des situations nouvelles (notamment simulées), qui mettent en présence du passé et du présent différents, hétérogènes et pluriels, on contribue à révéler à la fois le « pouvoir négatif d'inhibition du passé » (Lahire, 1998), de refoulement d'une partie des dispositions à agir liées aux situations antérieures, et en même temps on permet d'appréhender le potentiel d'activation de compétences, de manières de dire, de penser et de faire, laissées à l'état de veille jusqu'à ce jour (Leblanc, 2014b). En faisant réfléchir à l'efficacité professionnelle sous l'angle de sa double valence « objective » et « subjective» (Saujat, 2010), on favorise la recherche de compromis entre les normes personnelles de viabilité des acteurs relatives à la recherche de confort, d'efficience et de plaisir professionnels au niveau de l'usage de soi, et les normes prescrites par l'institution relatives aux objectifs de performance (Falzon, 2013 ; Ria, 2009 ; Saujat, 2010). Par exemple, pour faire face à des classes qui peinent à se mettre au travail, une activité écrite individuelle donnée aux élèves dès le début du cours, avec des consignes minimales, permet « objectivement» de les enrôler, de diminuer de manière efficace le bruit et de prendre en charge progressivement la classe. En offrant de nouvelles possibilités d'action aux enseignants (supervision de la classe, anticipation des comportements d'élèves...), cette façon de faire, discutable «objectivement» en termes de pertinence didactique, est vécue « subjectivement» comme leur donnant un grand confort d'enseignement et de la stabilité professionnelle au regard des premières expériences douloureuses (Ria \& Rouve, 2010).

\section{Conclusion}

L'état de l'art le plus récent sur la vidéoformation (Gaudin \& Chaliès, 2012) en pointe à la fois les effets bénéfiques en termes a) d'attractivité, b) de réflexivité, et c) d'évolution des préoccupations des enseignants débutants, mais également les zones d'ombre : autour du 
choix des vidéos et de qui les choisit, de l'agencement des objectifs de visionnage de vidéos au sein d'un programme de formation, de la nécessité d'un adossement théorique pour lire ces vidéos, et du lien entre les capacités d'interprétation développées lors de ces situations de vidéoformation et le développement du pouvoir d'agir en classe. Dans cette contribution, à partir du croisement de toutes les études menées actuellement sur les usages de la plateforme Néopass@ction, nous avons apporté des éléments de réflexion génériques sur les modalités d'acquisition de l'expérience professionnelle par la confrontation à des films et à des commentaires du travail réel d'autres enseignants en repérant et discutant les principaux effets transformatifs et les processus de transformation eux-mêmes mis à jour. D'autres études restent à mener dans des contextes de formation plus ordinaires avec des formateurs moins acculturés à la plateforme Néopass@ction et aux théories de l'activité afin de comprendre les difficultés d'appropriation de cet environnement mais aussi de repérer de nouveaux usages.

Dans le prolongement, il serait utile de mieux circonscrire et de valider par des études complémentaires les effets d'un dispositif d'analyse vidéo relatif à sa propre activité (Leblanc, 2014b) : a) mieux prendre en compte le vécu professionnel ; b) limiter la tendance des enseignants formés à se protéger et à masquer leurs difficultés (Linard \& Prax, 1984); c) réfréner la tendance des formateurs à porter un jugement normatif et à prescrire; et d) favoriser une démarche de co-enquête entre l'enseignant et le formateur pour accéder aux dimensions implicites de l'activité. L'articulation de ces deux dispositifs de vidéoformation « observer ses collègues » et «s'observer soi-même » serait à penser dans un continuum de professionnalisation envisagé sur une temporalité de plusieurs années (de la licence ou du master pré-professionnalisants aux premières années de classe).

\section{Références}

Blomberg, G., Stürmer, K., \& Seidel, T. (2011). How pre-service teachers observe teaching on video : Effects of viewers' teaching subjects and the subject of the video. Teaching and Teacher Education, 27(7), 1131-1140. doi : 10.1016/j.tate.2011.04.008

Citton, Y. (2012). Gestes d'humanités. Paris : Armand Collin.

Daniellou, F. (2002). Le travail des prescriptions. Conférence inaugurale du $37^{\mathrm{e}}$ Congrès de la SELF, Aix-en-Provence. Récupéré de http://www.ergonomie-self.org/documents/37emeAix-en-Provence-2002/daniellou.pdf

Dewey, J. (1993). Logique. La théorie de l'enquête. Paris : Puf. 
Durand, M. (2008). Un programme de recherche technologique en formation des adultes : une approche enactive de l'activité humaine et l'accompagnement de son apprentissage / développement. Éducation \& Didactique, 3(2), 97-121.

Falzon, P. (2013). Pour une ergonomie constructive. In P. Falzon (Ed.), Ergonomie constructive (pp. 1-15). Paris : Puf.

Flandin, S., \& Ria, L. (2012). Vidéo-formation des enseignants : apports de la didactique professionnelle. Actes $d u 2^{e}$ Colloque International de Didactique Professionnelle, Université de Nantes. Récupéré de http://tinyurl.com/didapro21

Flandin, S., \& Ria, L. (2014a). Un programme technologique basé sur l'analyse de l'activité réelle des enseignants débutants au travail et en vidéoformation. Activités, 11(2), 172-187.

Flandin, S., \& Ria, L. (2014b). Étude de l'activité d'un stagiaire d'EPS au travail et en vidéoformation autonome. Une « traçabilité » de l'évolution de l'intervention professionnelle. Recherches \& Éducations, 12, 57-73.

Flandin, S., \& Ria, L. (2014c). Comment les enseignants apprennent-ils de l'observation de leurs pairs? Une analyse de l'activité de vidéoformation en ligne de professeurs stagiaires. Journées Internationales d'EFTS, ConviSciencia de la recherche en éducation, Toulouse, 4-5 et 6 juin.

Flandin, S., \& Ria, L. (2014d). What do trainee teachers seek, see and learn with video ? Understanding the activity of trainee teachers when using a video-enhanced teacher-learning environment autonomously. Article soumis pour publication.

Gaudin, C., \& Chaliès, S. (2012). L'utilisation de la vidéo dans la formation professionnelle des enseignants novices : revue de littérature et zones potentielles d'étude. Revue française de pédagogie, 178, 115-130.

Gaudin, C., \& Chaliès, S. (à paraître). Video viewing in teacher education and professional development : A literature review. Educational Research Review.

Jullien, F. (2009). Les transformations silencieuses. Paris : Grasset.

Kersting, N.B., Givvin, K.B., Sotelo, F.L., \& Stigler, J.W. (2010). Teachers' analyses of classroom video predict student learning of mathematics : Further explorations of a novel measure of teacher knowledge. Journal of Teacher Education, 61, 172-181.

Lahire, B. (1998). L'homme pluriel. Les ressorts de l'action. Paris : Nathan.

Leblanc, S. (2014a). Vidéo formation et transformations de l'activité professionnelle, Activités, 11(2), 143-171. Récupéré de http://www.activites.org/v11n2/v11n2.pdf 
Leblanc, S. (2014b). Des dispositions concurrentes pour mener un entretien post-leçon : étude des effets d'un contexte d'entretien «innovant». In A. Muller \& I. Plazaola Giger (Eds), Dispositions à agir, travail et formation (pp. 53-75). Toulouse : Octarès.

Leblanc, S. (à paraître). Expériences mimétiques en vidéoformation et transformation de 1'activité professionnelle. Recherche et formation, 75.

Leblanc, S., \& Blanes, C. (2014). Immersion, réflexion, imagination et transformation via le visionnement de films de pairs. Journées Internationales d'EFTS, ConviSciencia de la recherche en éducation, Toulouse, 4-5 et 6 juin.

Leblanc, S., \& Ria, L. (2014). Designing the Néopass@ction platform based on modeling of beginning teachers' activity. Design and Technology Education : An International Journal, 19(2). 40-51.

Leblanc, S., Ria, L., Dieumegard, G., Serres, G., \& Durand, M. (2008). Concevoir des dispositifs de formation professionnelle des enseignants à partir de l'analyse de l'activité. @ctivités, 5(1), 58-78. Récupéré de http://activites.org/v5n1/leblanc.pdf

Leblanc, S., \& Sève, C. (2012). Vidéo formation et construction de l'expérience professionnelle. Recherche et formation, 70, 47-60.

Linard, M. (1989) Des machines et des hommes. Apprendre avec les nouvelles technologies. Paris : L'Harmattan.

Linard, M., \& Prax, I. (1984). Images vidéo, images de soi : Narcisse au travail. Paris : Dunod.

Lussi Borer, V., \& Muller, A. (2013). Analyse de l'activité, environnements de formation et développement professionnel. Différences intra et inter-activités : le possible, le réel et le virtuel. Conférence présentée au Colloque international «Enjeux actuels et futurs de la formation et de la profession enseignante en éducation », Montréal.

Lussi Borer, V., \& Muller, A. (2014a). Quel apport / usage du «voir» pour le « faire» en formation des enseignants du secondaire. In M. Altet, R. Etienne, L. Paquay \& P. Perrenoud (Eds), Comment la formation des enseignants prend-elle en compte la réalité du travail enseignant et les prescriptions dont il fait l'objet dans le milieu scolaire? (pp. 65-78). Bruxelles : De Boeck.

Lussi Borer, V., \& Muller, A. (2014b). Exploiter le potentiel des processus de renormalisation en formation à l'enseignement. Activités, 11(2), 129-142.

Lussi Borer, V., \& Muller, A. (à paraître). Connaître l'activité des enseignants en formation sur la plateforme Néopass@ction. Recherche et formation.

Peirce, C.S. (1978). Écrits sur le signe. Paris : Seuil. 
Quéré, L. (1993). Langage de l'action et questionnement sociologique. In P. Ladrière, P. Pharo, \& L. Quéré (Eds), La théorie de l'action. Le sujet pratique en débat (pp. 53-83). Paris : CNRS.

Ria, L. (2009). De l'analyse de l'activité des enseignants débutants en milieu difficile à la conception de dispositifs de formation. In M. Durand \& L. Filliettaz (Eds), Travail et formation des adultes (pp. 217-243). Paris : Puf.

Ria, L., \& Leblanc, S. (2011). Conception de la plateforme de formation Néopass@ction à partir d'un observatoire de l'activité des enseignants débutants : enjeux et processus. Activités, 8(2), 150-172. Récupéré de http://activites.org/v8n2/ria.pdf

Ria, L., \& Leblanc, S. (2012). Professionnalisation assistée par vidéo: les effets d'une navigation sur Néopass@ction. Recherches et Éducations, 7, 99-114.

Ria, L., \& Rouve, M.E. (2010). Observatoire du développement professionnel des néotitulaires en collèges «Ambition Réussite » : trajectoires, activités et identités. In R. Goigoux, L. Ria \& M.C. Toczek-Capelle (Eds), Les parcours de formation des enseignants débutants (pp. 255-269). Clermont-Ferrand : Presses universitaires Blaise Pascal.

Ricoeur, P. (1990). Soi-même comme un autre. Paris : Seuil.

Saujat, F. (2010). Travail, formation et développement des professionnels de l'éducation: voies de recherche en sciences de l'éducation (Note de synthèse pour l'HDR, non publiée, Université de Provence).

Schaeffer, J.M. (1999). Pourquoi la fiction ? Paris : Seuil.

Schwartz, Y. (2007). Un bref aperçu de l'histoire du concept culturel d'activité. @ctivités, 4(2), 122-133. Récupéré de http://www.activites.org/v4n2/v4n2.pdf

Seidel, T., Stürmer, K., Blomberg, G., Kobarg, M., \& Schwindt, K. (2011). Teacher learning from analysis of videotaped classroom situations : Does it make a difference whether teachers observe their own teaching or that of others ? Teaching and Teacher Education, 27(2), 259267.

Sensevy, G. (2012). Filmer la pratique : un point de vue de la théorie de l'action conjointe en didactique. In L. Veillard \& A. Tiberghien (Eds), Instrumentation de la recherche en éducation. Le cas du développement d'une base de vidéos de situation d'enseignement et d'apprentissage ViSA (pp. 5-62). Paris : Maison des sciences de l'Homme.

Sherin, M.G. (2001). Developing a professional vision of classroom events. In T. Wood, B.S. Nelson, \& J. Warfield (Eds), Beyond classical pedagogy: Teaching elementary school mathematics (pp. 75-93). Mahwah, NJ : Lawrence Erlbaum. 
Simondon, G. (2005). L'individuation à la lumière des notions de forme et d'information. Grenoble : Million.

Simondon, G. (2008). Imagination et invention. Chatou, France : La Transparence.

Steiner, P. (2010). Philosophie, technologie et cognition : état des lieux et perspectives. Introduction au dossier. Intellectica, 1(2), 7-40.

Stiegler, B. (2010). Philosopher par accident. Paris : Galilée.

Theureau, J. (2004). Cours d'action : méthode élémentaire. Toulouse : Octarès.

Theureau, J. (2010). Les entretiens d'autoconfrontation et de remise en situation par les traces matérielles et le programme de recherche «cours d'action». Revue d'anthropologie des connaissances, 4(2), 287-322. doi :10.3917/rac.010.0287

Varela, F.J. (1989). Autonomie et connaissance. Essai sur le vivant. Paris : Seuil.

Veillard, L. (2012). Les méthodologies de constitution et d'analyse des enregistrements données vidéo dans les recherches en éducation. In L. Veillard \& A. Tiberghien (Eds), Instrumentation de la recherche en éducation. Le cas du développement d'une base de vidéos de situation d'enseignement et d'apprentissage ViSA (pp. 123-161). Paris: Maison des sciences de l'Homme.

Zaccaï-Reyners, N. (2005). Fiction et typification. Contribution à une approche théorique de la transmission de l'expérience. Methodos, 5, 1-21. doi : 10.4000/methodos.378

Zhang, M., Lundeberg, M.A., Koehler, M.J., \& Eberhardt, J. (2011). Understanding affordances and challenges of three types of video for teacher professional development. Teaching and Teacher Education, 27(2), 454-462. 